\title{
The nodal structure of doubly-excited resonant states of helium
}

\author{
J M Rost $\dagger$, R Gersbacher $\dagger$, K Richter $\dagger$, J S Briggs $\dagger$ and D Wintgen $\ddagger$ \\ † Fakultät für Physik, Albert-Ludwigs-Universität, Hermann-Herder-Strasse 3, D-7800 \\ Freiburg, Federal Republic of Germany \\ ‡ Max-Planck-Institut für Kernphysik, Postfach 103980, D-6900 Heidelberg, Federal \\ Republic of Germany
}

Received 28 December 1990

\begin{abstract}
We examine the nodal structure of accurate helium wavefunctions calculated by direct diagonalization of the full six-dimensional problem. It is shown that for fixed interelectronic distance $R$ (or hyperspherical radius $\mathscr{R}$ ) the symmetric doubly-excited resonant states have well-defined $\lambda, \mu$ nodal structure indicating a near separability in prolate spheroidal coordinates. For fixed $\lambda$, however, a clear mixing of $R, \mu$ nodes is demonstrated. This corresponds to a breakdown of the adiabatic approximation and can be understood in terms of the classical two-electron motion.
\end{abstract}

\section{Introduction}

Evidence for approximate symmetries of doubly-excited resonant states of atoms has accumulated slowly over the past 25 years. As soon as it was recognized that these states represent a severe breakdown of the single-particle model (Fano 1983) alternative symmetries were proposed. The first of these was the 'plus-minus' classification of Cooper et al (1963) in which states of helium lying just below the $\mathrm{He}^{+}(N=2)+\mathrm{e}^{-}$ break-up threshold were described as linear combinations ( $2 \mathrm{~s} n \mathrm{p} \pm 2 \mathrm{p} n \mathrm{~s})$. Later Wulfman and Sukeyuki (1973) and Sinanoglu and Herrick (1973) began a search for internal symmetries based on a reduction of the $\mathrm{SO}_{4} \otimes \mathrm{SO}_{4}$ group classification of the noninteracting two-electron problem. This approach was examined exhaustively by Herrick and co-workers in a series of papers (Herrick and Sinanoglu 1975, Herrick 1975, Herrick and Kellman 1980, Herrick et al 1980, Kellman and Herrick 1980) the results of which are summarized in a major review article by Herrick (1983). These results set the framework for much subsequent analysis of symmetry properties, based either on an empirical examination of calculated wavefunctions or on model Hamiltonians (Lin and Macek 1984, Ho and Callaway 1984, Komninos and Nicolaides 1986, Yuh et al 1981, Makrewicz 1989).

The important results of Herrick's analysis may be summarized as follows.

(i) Each state in a given $N$ manifold can be assigned internal quantum numbers $(K, T, A)$. The quantum number $K$ was shown to characterize the expectation value of $\cos \Theta$, where $\Theta$ is the angle between the vectors $r_{1}, r_{2}$ of the two electrons with respect to the nucleus. The quantum number $T$ arises from quantizing the projection $\boldsymbol{L} \cdot \hat{\boldsymbol{B}}$ of the total angular momentum vector along the direction of $\boldsymbol{B}=\boldsymbol{b}_{1}-\boldsymbol{b}_{2}$, the difference of the independent-electron Runge-Lenz vectors $b_{1}, b_{2}$. The quantum number 
$A= \pm 1$ describes the even or odd symmetry of the wavefunction with respect to the line $r_{1}=r_{2}$ and reflects the Pauli principle.

(ii) Sequences of intrashell resonant states (i.e. both electrons with the same principal quantum number $N$ ) of different spin $S$, total angular momentum $L$ and parity $\pi$ may be grouped into 'rotor' series.

(iii) States can also be assigned a 'vibrational' quantum number $n_{v}=$ $\frac{1}{2}(N-K-T-1)$. This latter property emphasizes one weakness of Herrick's analysis, namely, that the quantum numbers are not all independent of each other.

In addition to (iii) one has $A=\pi(-1)^{S+T}$.

An ostensibly completely different approach to the symmetry of doubly-excited resonances was proposed by Feagin and Briggs $(1986,1988)$ based on earlier work on singly excited states (Hunter and Pritchard 1967). In this method the interelectronic axis $\boldsymbol{R}=\boldsymbol{r}_{\mathbf{1}}-\boldsymbol{r}_{2}$ is treated as an adiabatic 'molecular' axis in analogy to the internuclear axis in diatomic molecules (the important point is that the adiabatic axis is that between particles having a repulsive interaction). In the molecular approach the following symmetries of doubly excited states emerge (Feagin and Briggs 1986, 1988, Rost et al 1990, Rost and Briggs 1990).

(i) In the body fixed frame the interelectronic centre-of-mass (ЕCM) motion described by $\boldsymbol{r}=\left(\boldsymbol{r}_{1}+\boldsymbol{r}_{2}\right) / 2$ is separable for fixed $R$. The separation is in prolate spheroidal coordinates $\lambda, \mu, \varphi$ and the associated quantum numbers are the molecular quantum numbers $n_{\lambda}, n_{\mu}, m$, where the latter denotes the projection of the ECM angular momentum and the total angular momentum along the body-fixed axis $\hat{R}$.

(ii) Rotational structure appears naturally in the molecular model and the rotor series of Herrick are sequences of states of different $L$ and $S$ built on a single mo.

(iii) The gerade-ungerade character of the mo is a preserved symmetry of the full problem according as $\pi(-1)^{S}$ is even or odd.

(iv) The spheroidal nodal surfaces become spherical or paraboloidal in the limits $R \rightarrow 0$ and $R \rightarrow \infty$ respectively: The number of nodal surfaces is conserved for all $R$.

In the latter series of papers it has been shown also that the independent mo quantum numbers $n_{\lambda}, n_{\mu}, m$ contain and explain the Herrick quantum numbers $K, T$, $A$ and $n_{v}$. The latter quantum numbers are simply combinations of the parabolic quantum numbers describing a polarized state in the single-electron $N$-manifold resulting from the removal of one electron to infinity whilst the other remains bound. These parabolic nodal surfaces, with quantum numbers $n_{1}, n_{2}, m$, are conserved as spheroidal surfaces for finite $R$ and give the connection

$$
\begin{aligned}
& A=(-1)^{n_{\mu}} \\
& n_{v}=n_{\lambda}=n_{1} \\
& T=m \\
& K=\left(n_{\mu}-(1-A) / 2\right) / 2-n_{\lambda} \equiv n_{2}-n_{1} .
\end{aligned}
$$

Furthermore the quantum number $K$ can also be associated with the asymptotic $(R \rightarrow \infty)$ value of the separation constant in the $\lambda, \mu$ equations; the separability itself being due to the presence of an extra constant of the motion connected with the $\mathrm{SO}_{4}$

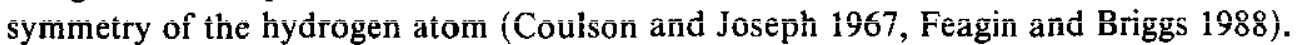

The validity of the approximate mo separability has been only indirectly supported until recently. For example mo potential curves, scaled from those of $\mathrm{H}_{2}^{+}$, show a striking similarity (Feagin and Briggs 1986) to hyperspherical (HS) potential curves calculated numerically by a diagonalization in five space dimensions still under an 
adiabatic assumption regarding the hyper-radius. The (KTA) quantum numbers empirically assigned to the HS curves (Lin 1984) correspond exactly to the Mo designation according to (1). Propensity rules arising from the mo classification of states (Rost and Briggs 1990, hereafter referred to as $R_{B}$ ) have been shown to explain relative transition rates. It has also been shown that the adiabatic mo energies become accurate for high-lying states (Rost 1990). Recently convincing new evidence for the MO separability has emerged. A calculation of $\mathrm{HS}$ wavefunctions for the $\left(N^{1} \mathrm{P}^{\circ}\right)$ intrashell resonant sequence in $\mathrm{H}^{-}$has been performed by diagonalization of the adiabatic hyperspherical problem in a large basis set (Sadeghpour and Greene 1990). The contour plots of this wavefunction for fixed hyper-radius $\mathscr{R}=\left(r_{1}^{2}+r_{2}^{2}\right)^{1 / 2}$ show well pronounced nodal surfaces which have been shown to agree exactly in number and shape with those to be expected from a $\lambda, \mu$ separability (Rost et al 1991). Until now, however, a similar analysis of accurate wavefunctions from a full diagonalization of the sixdimensional problem has been lacking. Here we present for the first time such an analysis. The conclusion is that numerically accurate intrashell resonant wavefunctions (Gersbacher and Broad 1990) also show the $\lambda, \mu$ nodal structure indicating nearseparability. The separability is not complete, however, and particularly there appears a mixing of the $R$ and the $\mu$ motions. This is shown to be due to a breakdown of the adiabatic assumption. In addition, the nodal structure of the two-electron wavefunctions will be shown to be connected intimately with the occurrence of a near-stable periodic orbit in the classical mechanics of the Coulomb three-body problem. This is a first indication that the structure of the three-body resonant states will reflect the underlying classical mechanics in exactly the same way as has now been demonstrated in great detail in the case of a hydrogen atom in a magnetic field (Friedrich and Wintgen 1989, Hasegawa et al 1989).

\section{The spheroidal nodal structure}

In terms of single-electron coordinates in the body-fixed frame the spheroidal coordinates are

$$
\begin{aligned}
& \lambda=\left(r_{1}+r_{2}\right) / R \\
& \mu=\left(r_{1}-r_{2}\right) / R
\end{aligned}
$$

and the associated nodal surfaces are ellipsoids and hyperboloids of revolution respectively about the interelectronic axis. In the case of ${ }^{1} \mathrm{P}^{\circ}$ symmetry Sadeghpour and Greene (1990) have calculated Hs 'inner' wavefunctions $\Phi(\alpha, \Theta, \mathscr{R})$ in the two variables $\alpha=\tan ^{-1}\left(r_{1} / r_{2}\right), \Theta=\cos ^{-1}\left(\hat{\boldsymbol{r}}_{1} \cdot \hat{\boldsymbol{r}}_{2}\right)$ for fixed hyper-radius $\mathscr{R}$ for the intrashell sequences with principal quantum number $N \leqslant 11$. The density of the lowest two members of the $N=6$ manifold for $\mathscr{R}=80$ au (roughly the position of maximum probability in $\mathscr{R}$ ) are shown in figure 1 . Clearly the nodal lines do not correspond to $\alpha$ or $\Theta$ nodes, i.e. $\alpha=$ constant or $\Theta=$ constant. However, a simple mapping

$$
\lambda, \mu=\frac{1 \pm \tan \alpha}{\left(1+\tan ^{2} \alpha-2 \tan \alpha \cos \Theta\right)^{1 / 2}}
$$

(where the + sign denotes $\lambda$ and the - sign denotes $\mu$ ) allows the nodal lines $\lambda=$ constant and $\mu=$ constant to be overdrawn in the plot of figure 1 and these nodal lines show almost perfect agreement with the calculation (Rost et al 1991). Not only that, the number of nodal lines shows that the states are built on the $10 m \pi_{u}$ mo with 

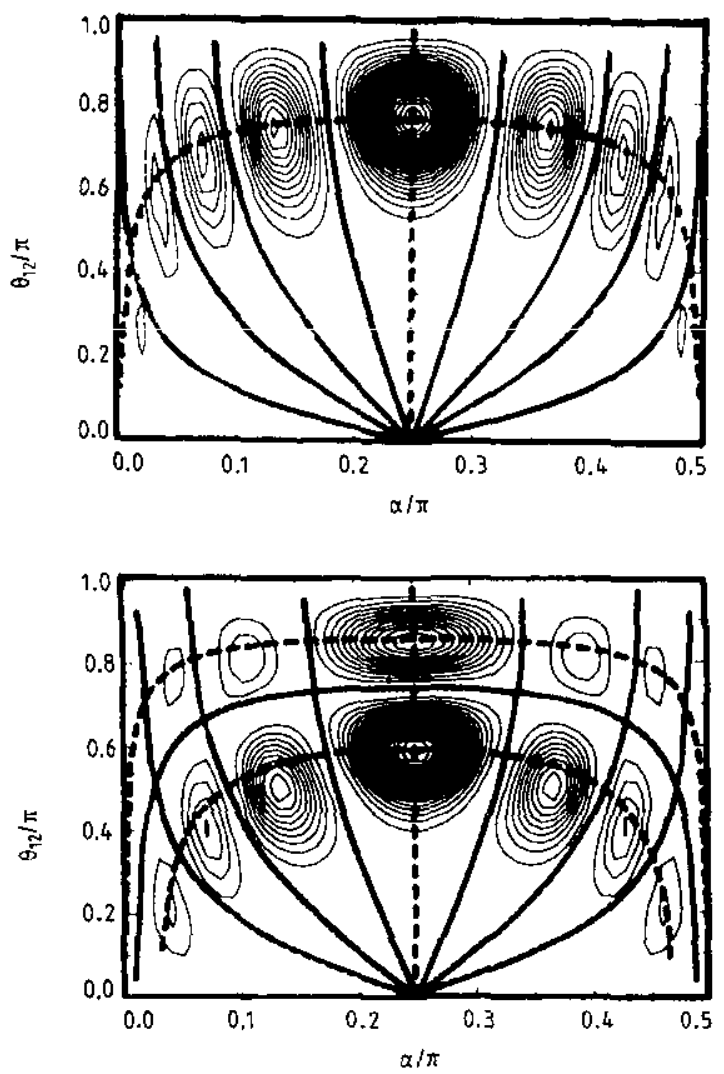

Figure 1. The contour plot of ${ }^{1} \mathrm{p}^{\circ}$ adiabatic two-electron densities in the $N=6$ manifold at $\mathscr{R}=80$ au taken from Sadegphour and Greene (1990). The nodal (continuous) and antinodal (dashed) lines in $\lambda, \mu$ coordinates for $(a)$ the state with $\left(n_{\lambda} n_{\mu} m\right)=(081)$ and (b) the state with $\left(n_{\mathrm{A}} n_{\mu} m\right)=(161)$.

$n_{\lambda}=0, n_{\mu}=8, m=1$ (figure $1(a)$ ) and the $9 l \pi_{i}$ MO with $n_{\lambda}=1, n_{j}=6, m=1$ (figure $1(b))$. When translated into $K, T$ and $A$ according to (1) the resulting assignment agrees with that of Sadeghpour and Greene (1990).

Although the mo nodal structure of the adiabatic HS wavefunction is striking, Sadeghpour and Greene assumed that the single-channel adiabatic approximation is valid and justified their assumption by the close agreement of their calculated energies with other calculations and with experiment. Despite this agreement, it is not clear to what extent the near-separability demonstrated in figure 1 depends upon the adiabatic assumption.

Therefore we consider it necessary to compare with completely independent, full diagonalization wavefunctions to test the $\lambda, \mu$ separability.

Gersbacher and Broad (1990) have calculated accurate wavefunctions for low-lying doubly-excited states by direct diagonalization in a Sturmian basis based upon independent-electron coordinates $\boldsymbol{r}_{1}, \boldsymbol{r}_{2}$. The accuracy of their calculation is indicated by close agreement not only with measured resonance energies but also with the detailed shape of the Fano profile (Kossmann et al 1988). Accordingly we have taken the wavefunction of Gersbacher and Broad (1990) and plotted their probability density in various ways by keeping certain variables constant. The results, shown in figures $2-5$ confirm the 
near-separability of the complete wavefunction $\Psi\left(\boldsymbol{r}_{1}, \boldsymbol{r}_{2}\right)\left({ }^{2 S+1} L^{\pi}\right)$ in $\lambda, \mu$ internal coordinates.

The first example is the lowest $N=4{ }^{1} \mathrm{P}^{0}$ intrashell resonance. This is of $\pi(-1)^{S}=$ odd, i.e. ungerade mo character built on the $n_{\lambda}=0, n_{\mu}=4, m=1$ (parabolic. $n_{1}=0$, $n_{2}=2, m=1, A=+1$ and therefore $K, T, A=2,1,1$ ) Mo $6 \mathrm{~h} \pi_{\mathrm{u}}$. In figure 2 the probability density

$$
P(\mathscr{R}, \Theta, \alpha)=|\Psi|^{2} \mathrm{~d} V(\mathscr{R}, \Theta, \alpha)
$$

is plotted for fixed hyper-radius $\mathscr{R}$ and averaged over Euler angles relating the space-fixed to the body-fixed frame, to compare directly with the hyperspherical adiabatic density of figure 1 . One sees that the nodal structure of this accurate wavefunction is of the same character as the HS wavefunction of figure 1 . The nodes agree in number and shape with the $\left(n_{\lambda} n_{\mu} m\right)=(041)$ classification, i.e. the $6 \mathrm{~h} \pi_{\mathrm{u}}$ Mo.

The next example is the lowest $N=3^{\prime} S^{\mathrm{e}}$ intrashell resonance. This state has $\pi(-1)^{S}=+1$ and therefore is of gerade character and based on the $5 \mathrm{~g} \sigma_{\mathrm{g}}$ mo with $n_{\lambda}=0, n_{\mu}=4, m=0$. The probability density of this state is shown in figure 3 for fixed hyper-radii $\mathscr{R}=8$ and $\mathscr{R}=6$ au again as a function of $\alpha$ and $\Theta$ to compare with the plots of internal Hs wavefunctions (figure 1). The close similarity is evident and provides support for the adiabatic picture in a body-fixed frame. Again the number of nodes $n_{\lambda}=0, n_{\mu}=4$ confirm the molecular coordinate separation. Such a symmetric 'in-saddle' state $(\mathrm{RB})$ should have its maximum density on the saddle $\left(r_{1} \approx r_{2}, \tan \alpha \approx \pi / 4\right)$ at a radius $\mathscr{R} \approx 8$ au and this is seen clearly in figure $3(b)$. At smaller hyper-radius, density shifts off the saddle as shown in figure $3(a)$ but still the $\lambda, \mu$ nodal structure is preserved.

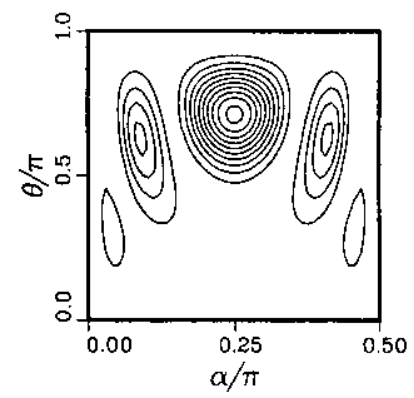

Figure 2. The two-electron density for the ' $\mathrm{P}^{\circ} N=4$ resonance with $\left(n_{\lambda} n_{\mu} m\right)=(041)$ at $\mathscr{R}=18$ au from full diagonalization.
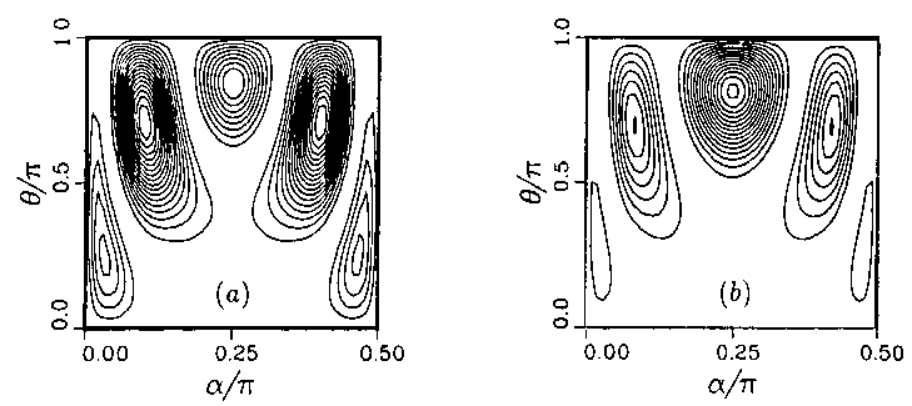

Figure 3. Same as in figure 2 but for the ' $\mathrm{S}^{\mathrm{e}} N=3$ resonance with $\left(n_{\lambda} n_{\mu} m\right)=(040)$ : (a) at $\mathscr{R}=6$ au and $(b)$ at $\mathscr{R}=8$ au. 
This is emphasized in figure $4(a)$ where the same densities are plotted against $\lambda, \mu$ for fixed hyper-radius. As expected the nodal lines are perpendicular to the $\mu$ axis.

Although the HS adiabatic wavefunction shows $\lambda, \mu$ nodal structure, the adiabatic HS Hamiltonian is not directly separable in $\lambda, \mu$ coordinates. The adiabatic mo Hamiltonian with electron separation $R$ fixed is separable, however. Therefore in figure $4(b)$ the exact probability density of the lowest $N=3^{1} S^{\mathrm{e}}$ intrashell resonance is shown in molecular coordinates, where the interelectronic axis $R$ is held fixed and the density is plotted as a function of $\lambda$ and $\mu$. Note that the hyperspherical radius $\mathscr{R}$ can be rewritten $\mathscr{R}=\left(4 r^{2}+R^{2}\right)^{1 / 2} / \sqrt{2}$ and since, in the states plotted in figures $1-5$, the ECM motion is strongly localized, i.e. $r \ll R$, then $\mathscr{R} \approx R / \sqrt{2}$. Hence a plot at constant $\mathscr{R}$ is almost a plot at constant $R$ which is clearly seen from figure 4 . In a single-channel Mo adiabatic calculation, wavefunctions at constant $R$ show, by definition, $\lambda, \mu$ separability. The electron densities from the mo wavefunctions (figure $4(c)$ ) agree qualitatively with the exact contours of figure $4(b)$.

In figure $5(a)$ we show plots of the probability density for fixed $R$ of the second ${ }^{1} \mathrm{~S}$ e intrashell resonance below the $N=3$ threshold. This state is excited in the bending mode, i.e. has $n_{\lambda}=1$ and the residual excitation is in the $\mu$ direction with $n_{\mu}=2$. The internal Mo is therefore the $4 \mathrm{~d} \sigma_{\mathrm{g}}$ state which is a member of the $2 \mathrm{~s} \sigma_{\mathrm{g}}, 4 \mathrm{~d} \sigma_{\mathrm{g}}, 6 \mathrm{~g} \sigma_{\mathrm{g}} \ldots$ insaddle sequence ( $\mathrm{RB}$ ). The nodal lines are almost perfect straight lines now in $\mu$ and $\lambda$ as can be seen in figure $5(a)$. This behaviour is expected from the adiabatic mo density (figure $5(b)$ ) which again agrees qualitatively with the exact contours of figure $5(a)$.
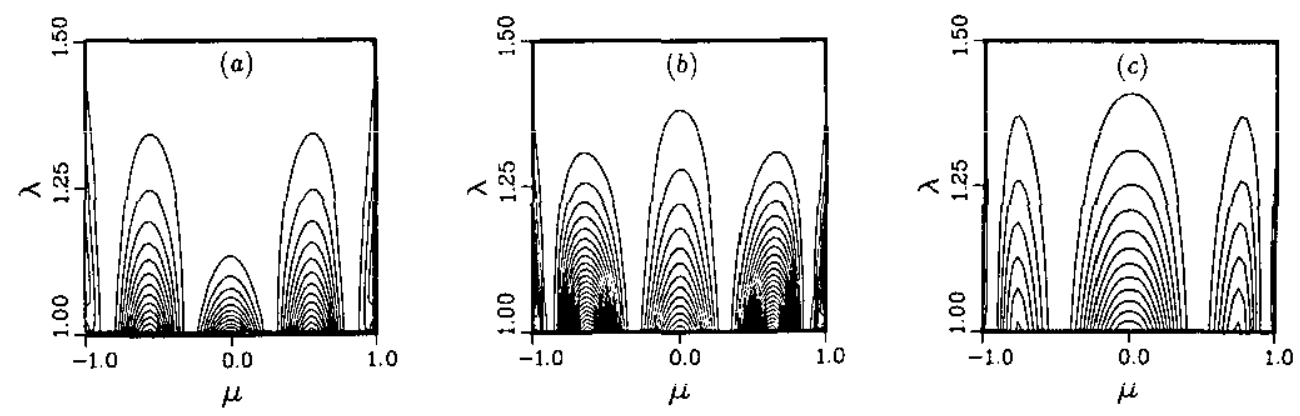

Figure 4. The same state as in figure 3 but in $\lambda, \mu$ coordinates. The density $(a)$ from full diagonalization at fixed hyper-radius $\mathscr{R}=6$ au and $(b)$ at fixed interelectronic distance $R=9$ au, $(c)$ from the adiabatic MO approximation at $R=9$ au.
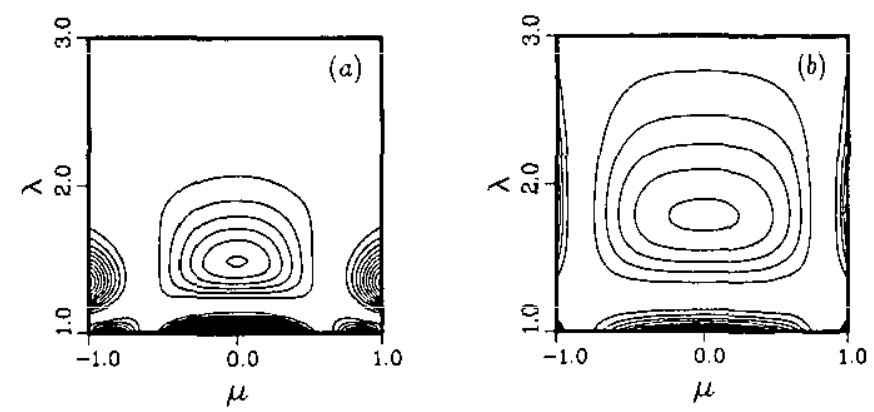

Figure 5. The ' $\mathrm{S}^{\mathrm{e}} N=3$ resonance with $\left(n_{\lambda} n_{\mu} m\right)=(120)$ at $R=8$ au ( $\left.a\right)$ from full diagonalization and $(b)$ in the Mo approximation. 
In summary, for the first time we have shown that accurate numerically-calculated six-dimensional wavefunctions for the intrashell resonant states of helium show a nodal structure indicating an approximate separability in mo $\lambda, \mu$ coordinates.

\section{Adiabatic and diabatic motion}

The accuracy of the calculated single-channel hyperspherical resonance energies (Sadeghpour and Greene 1990) presented in section 2 would indicate that the adiabatic treatment in $\mathscr{R}$ or $R$ is essentially valid. However, it has also been shown (Rost and Briggs 1988, 1989) that a single diabatic potential curve connecting 'saddle sequences' of mo can be used to calculate, in an extremely simple way, accurate resonant energies for complete Rydberg sequences of doubly excited states. In the adiabatic description, each state of the sequence of lowest intrashell resonances in a manifold $N$ is built on a given internal mo motion. For the $N=3$ state considered in section 2 this was the $5 \mathrm{~g} \sigma_{\mathrm{g}}$ MO, the third member of the $1 \mathrm{~s} \sigma_{\mathrm{g}}, 3 \mathrm{~d} \sigma_{\mathrm{g}}, 5 \mathrm{~g} \sigma_{\mathrm{g}}, 7 \mathrm{i} \sigma_{\mathrm{g}}, \ldots$, in-saddle series shown in figure 6. The resonant state would be the lowest (nodeless) vibrational state in the $5 \mathrm{~g} \sigma_{\mathrm{g}}$ potential well. Hence the corresponding total wavefunction should have four nodes in $\mu$ and zero nodes in $R$. This corresponds to an asymmetric stretch motion of the ECM over the saddle point of the adiabatic potential (see figures $6(c)$ and $6(d)$ of Rost and Briggs 1989). By contrast the resonant states in the pure diabatic potential (which has the nodal structure of the lowest mo of the saddle sequence, in the example considered here, the nodeless $1 \mathrm{~s} \sigma_{\mathrm{g}}$ MO with $n_{\lambda}=n_{\mu}=m=0$ ) have increasing number of $R$ nodes. This motion corresponds to a symmetric stretch in which the ECM sits on the saddlepoint $r_{1} \approx-r_{2}$.

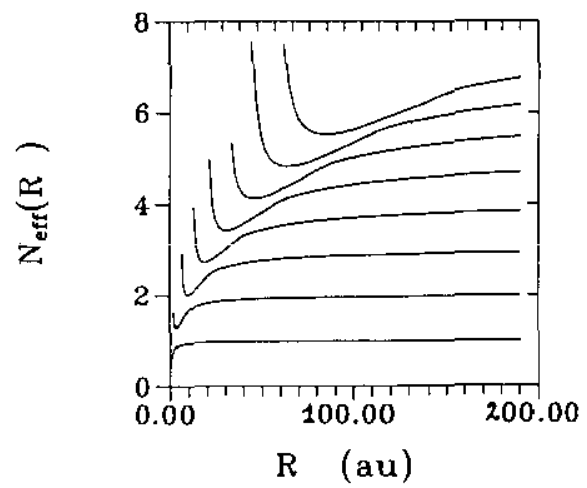

Figure 6. The in-saddle sequence of adiabatic MO $1 \mathrm{~s} \sigma_{\mathrm{g}}, 3 \mathrm{~d} \sigma_{\mathrm{g}} \ldots$ with $\left(n_{\lambda}, n_{\mu}, m\right)=$ $(0,2(N-1), 0)$. The adiabatic potential energy is in units of $N_{\text {eff }}=\sqrt{-2 / U(R)}$.

The adiabatic and diabatic pictures correspond to the two extreme limits of infinitely slow or infinitely fast $R$ motion in the region of the avoided crossings. Both pictures give accurate values of the resonant positions approximated as bound states. Clearly neither is correct since the resonances are known to decay. It is also clear that decay of the state is connected intimately with the $R$ or $\mu$ motion parallel to the direction of negative curvature at the saddle point. We demonstrate in figure 7 the fragility of $\mu$ nodes with plots of the probability density of the lowest ' $S^{e} N=4$ intrashell resonance, the fourth member of the in-saddle series of figure 6 . The state built on the $7 \mathrm{i} \sigma_{\mathrm{g}}$ MO 

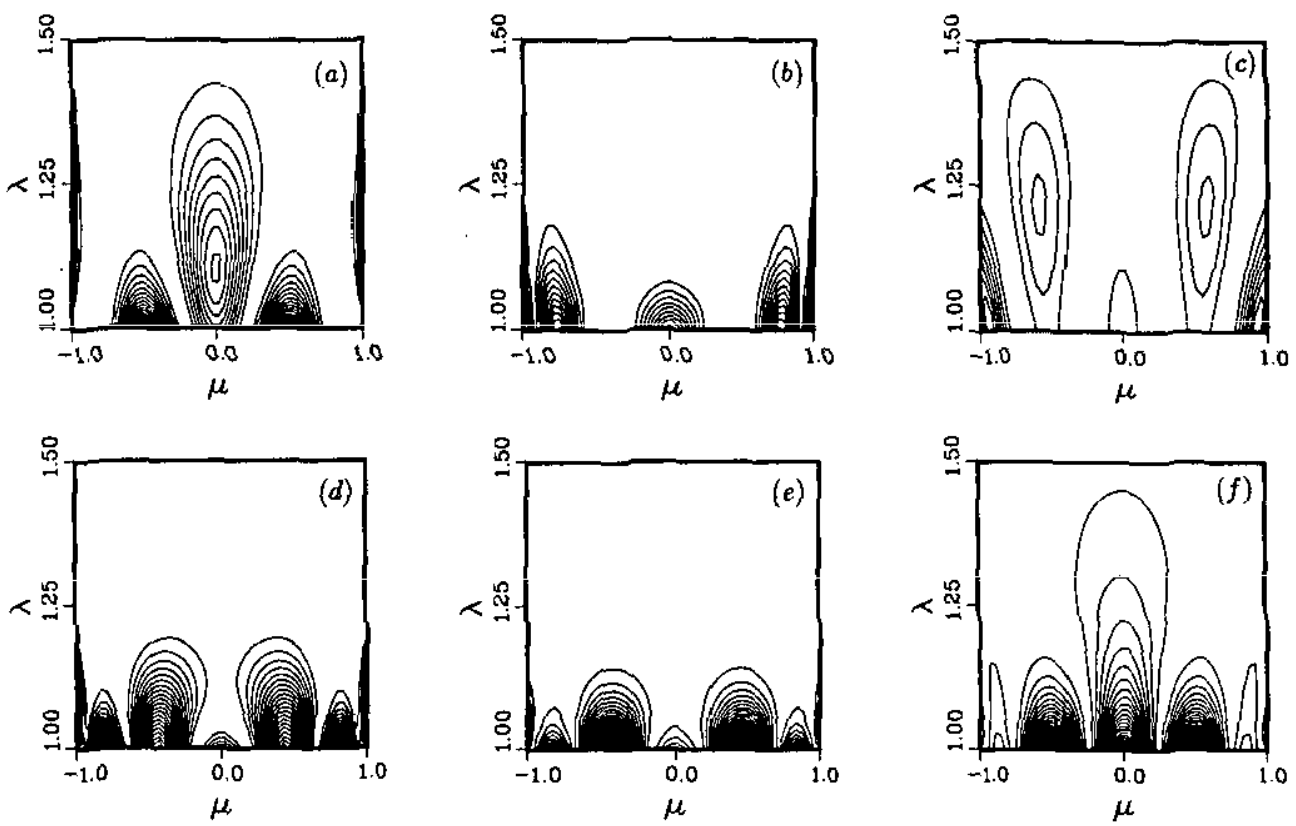

Figure 7. The ' $S^{e} N=4$ resonance with $\left(n_{\lambda} n_{\mu} m\right)=(060)$ from full diagonalization at $R=9$ au $(a), R=12$ au $(b), R=13$ au $(c), R=15$ au $(d), R=17$ au $(e)$ and $R=20$ au $(f)$.

with $\left(n_{\lambda} n_{\mu} m\right)=(060)$ has six $\mu$ nodes. They can be identified on the figures $7(d-f)$ which show the contours in the region $R \approx 20$ where the resonance is localized. But for smaller $R$ values (figures $7(a-c)$ ) only four $\mu$ nodes are present. The obvious change in the number of $\mu$ nodes is expected when the avoided crossing (figure 7 ) between the $5 \mathrm{~g} \sigma_{\mathrm{g}}$ MO $(040)$ and the $7 \mathrm{i} \sigma_{\mathrm{g}} \mathrm{MO}(060)$ is traversed diabatically. The diabatic behaviour of the exact wavefunction in the region of avoided crossings couples $R$ and $\mu$ motion.

By contrast, bending vibration connected with the excitation along the direction of positive curvature at the saddle point, i.e. in $\lambda$ (figure $6(b)$ of Rost and Briggs 1989), couples weakly to decay. Accordingly the number of $\lambda$ nodes is preserved for all $R$. This observation is the origin of the decay propensity rules of $R B$. The second ${ }^{1} S^{e} N=3$ intrashell resonance with nodal structure (120) arising from the $4 \mathrm{~d} \sigma_{\mathrm{g}}$ MO has already been shown in figure 5. The additional plots of figure 8 indicate the change of $\mu$ nodes due to the avoided crossing between the members $2 \mathrm{~s} \sigma_{\mathrm{g}}(100)$ and $4 \mathrm{~d} \sigma_{\mathrm{g}}(120)$ of the
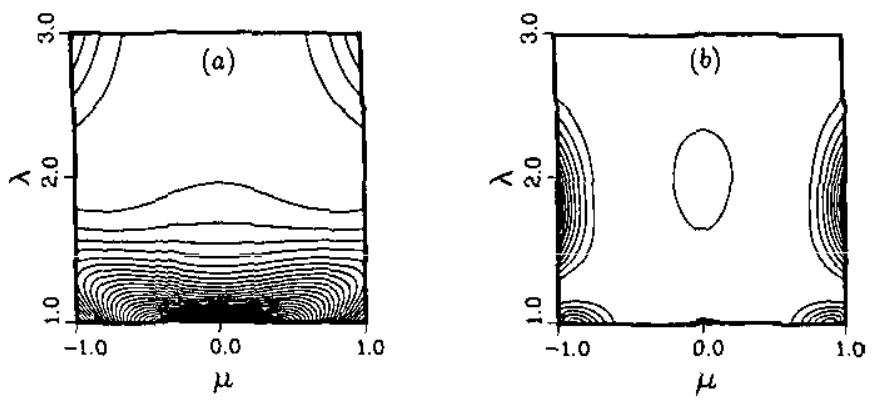

Figure 8. Same as in figure $5(a)$ but $(a)$ at $R=1$ au and $(b)$ at $R=5$ au. 
corresponding saddle sequence. For smaller $R$ values (figure $8(a)$ ) the two $\mu$ nodes disappear but the single $\lambda$ node remains. This is again the expected behaviour when the avoided crossing is traversed diabatically.

\section{Classical periodic orbits}

Recently, periodic orbits have been used to analyse complex spectra (Friedrich and Wintgen 1989) and to understand the dynamical localization of wavefunctions (Heller 1984). The classical mechanics of the three-body atomic Coulomb problem has been studied quantitatively by Klar (1986), by Poirier (1989) and by Richter and Wintgen (1990). In figure 9 a collinear periodic orbit is shown underlying the major part of the electron probability distribution of the lowest $N=4{ }^{1} \mathrm{~S}^{\mathrm{e}}$ intrashell resonance in helium (see also figure 7). The trajectory is analogous to the asymmetric stretch mode in molecules and corresponds to localization of the wavefunction in the $R, \mu$ plane as can be seen in figure 9 . It is only weakly unstable and this is due to the relatively large interelectronic separation maintained along this orbit. In contrast the bending vibration is classically stable. Excitation of this mode would quantum mechanically result in $\lambda$ nodes; see for example figure 5.

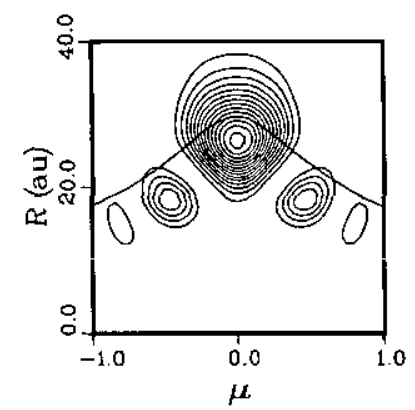

Figure 9. Same as in figure 7 but for fixed $\lambda=1.01$ in the $(R, \mu)$ plane. The continuous line is a collinear classical periodic orbit scaled to the resonance energy (see text).

The separate behaviour of $r_{1}(t), r_{2}(t)$ in this periodic orbit is shown in figure 10 . When one electron is far from the nucleus, the other is very close as would be obtained in an asymmetric stretch mode, i.e. the electrons oscillate out of phase. Hence although one electron is close to the nucleus (and hence the ECM is way off the saddle point) the orbit does not decay since the interelectronic distance is large and energy not readily exchanged. That the orbit is not pure asymmetric stretch is also clear. Such a mode would correspond to constant hyperspherical radius which is not the case for the orbit shown in figure 9 . Nevertheless in the region $r_{1} \approx r_{2}$ near the saddle point the ECM has the lowest velocity within a period (figure $10(b)$ ) and it is this feature that results in a maximum of probability of the state on the Wannier saddle. Note that this orbit bears no resemblance to the symmetric stretch motion $\left(r_{1}(t)=r_{2}(t)\right)$ along the Wannier ridge with which the lowest intrasheli resonances have been associated for a long time (Fano 1983). Similar conclusions have been drawn by Ezra (1990) based on studies of a collinear helium model.

Remarkably one sees in figure 9 that nodal lines are approximately diagonal in the $R, \mu$ plane. A rotation of the axes about $45^{\circ}$ would give a nodal pattern parallel to 

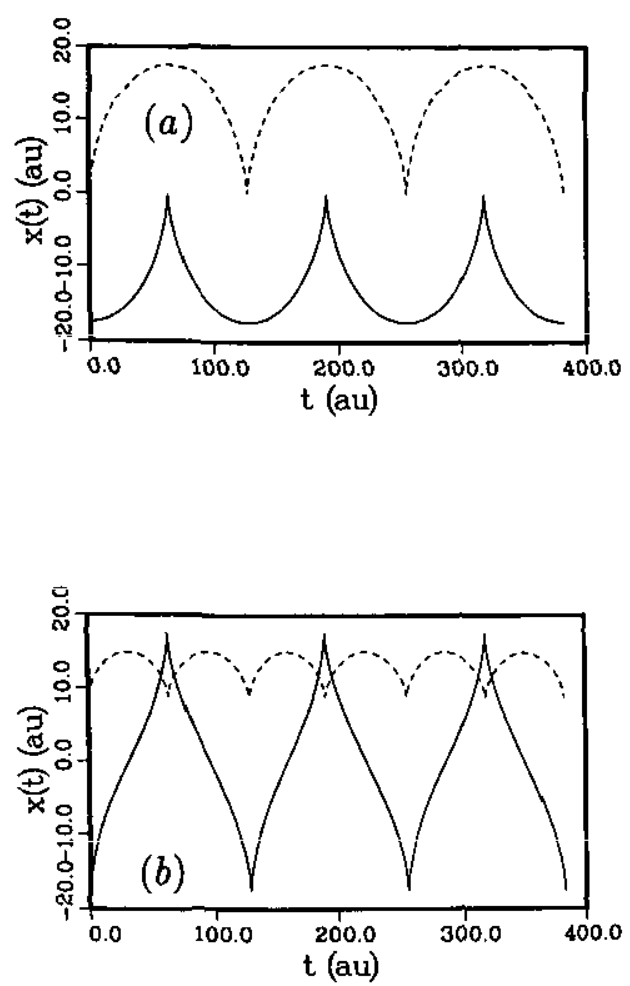

Figure 10. The time dependence of vectors of the classical periodic orbit from figure 9: (a) the single-electron vectors $x(t)=r_{i}, i=1,2$ and $(b)$ the molecular coordinates $x(t)=r(t)$ of the ECM (solid) and $\boldsymbol{x}(i)=\boldsymbol{R}(i)$ of the intereiectronic axis (dashed).

the new axes $x, y=R(1 \pm \mu)$. These coordinates have been known for decades in the two-electron problem (James and Coolidge 1937) and were already used by Pekeris (1958) for the ground states of the helium isoelectronic sequence. Presently they are used in a new computer code for the direct calculation of highly doubly-excited states (Wintgen 1991). The equal mixture of $\hat{K}$ and $\mu$ nodes in figure $\hat{9}$ again emphasizes that the nodal structure of numerical full diagonalized wavefunctions differs from that derived within an adiabatic approximation as discussed in section 3 . On the other hand the interelectronic velocity $(\mathrm{d} / \mathrm{d} t) R(t)$ is on average much smaller than the ECM velocity $(\mathrm{d} / \mathrm{d} t) r(t)$ (figure $10(b)$ ). This may explain why these intrashell resonances can be treated adiabatically in $R$ or $\mathscr{R}$ to good approximation.

\section{Conclusions}

We have examined the nodal structure of numerically exact wavefunctions for intrashell doubly excited states of ${ }^{1} S^{e}$ and ${ }^{1} P^{o}$ symmetry. We have shown that for fixed interelectronic separation $R$ the wavefunctions show an almost pure $\lambda, \mu$ nodal pattent. This has also been shown previously (Rost et al 1991) for adiabatic HS wavefunctions plotted at constant hyper-radius $\mathscr{R}$. However, when $\lambda$ is held constant one sees that the $R$ and $\mu$ nodal structures are not pure. This proves that the bending vibration, if described in the coordinate $\lambda$, separates from motion in the plane of asymmetric and 
symmetric stretch modes. The mixing of the latter modes, corresponding in $R, \mu$ coordinates to the avoided crossings between adiabatic mo potentials (figure 6), implies that neither a full adiabatic nor a full diabatic motion describes the complete structure of intrashell states. The reason that both pictures have led to the calculation of accurate energies lies in the fact that in both approximations the maximum probability density occurs near $r_{1} \approx r_{2}$ as in the exact wavefunctions. This indicates that the energy eigenvalue alone is not a particularly sensitive test of the goodness of a wavefunction.

It has been shown that the exact wavefunction for fixed $\lambda=1$ has high probability along a particular collinear classical periodic orbit. In this orbit the electrons oscillate out of phase such that when one is close to the nucleus the other is near the outer turning point but they spend a considerable fraction of the orbital period near $r_{1} \approx r_{2}$ consistent with the build up of probability density of wavefunctions in this region.

The major result of this work is that although for fixed $R$ or $\mathscr{R}$ the wavefunctions have $\lambda, \mu$ nodal structure over a wide range of $R$, there is no separability in $R$ and $\mu$ for fixed $\lambda$. This suggests that the known adiabatic single-channel wavefunctions for doubly excited intrashell states are not a good approximation to the true wavefunction in the whole configuration space.

\section{References}

Cooper J W, Fano U and Prats F 1963 Phys. Rev. Lett. 10518

Coulson C A and Joseph A 1967 Int. J. Quantum Chem. 1337

Ezra G S 1990 private communication

Fano U 1983 Rep. Prog. Phys. 4697

Feagin J M and Briggs J S 1986 Phys. Rev. Lett. 57984

1988 Phys. Rev. A 374599

Friedrich H and Wintgen D 1989 Phys. Rep. 18337

Gersbacher R and Broad J T 1990 J. Phys. B: At. Mol. Opt. Phys. 23365

Hasegawa H, Robnik M and Wunner G 1989 Prog. Theor. Phys. 98198

Heller E J 1984 Phys. Rev. Lett. 531515

Herrick D R 1975 Phys. Rev. A 12413

1983 Adv. Chem. Phys. 521

Herrick D R and Kellman M E 1980 Phys. Rev. A 21418

Herrick D R, Kellman M E and Poliak R D 1980 Phys. Rev. A 221517

Herrick D R and Sinanoglu O 1975 Phys. Rev. A 1197

Ho Y K and Callaway J 1984 J. Phys. B: At. Mol. Phys. 17 L559

Hunter G and Pritchard H O 1967 J. Chem. Phys. 462153

James H M and Coolidge A S 1937 Phys. Rev. 51857

Kellmann M E and Herrick D R 1980 Phys. Rev. A 221536

Klar H 1986 Phys. Rev. Lett. 5766

Komninos Y and Nicolaides C A 1986 J. Phys. B: At. Mol. Phys. 191701

Kossmann H, Krässig B and Schmidt V 1988 J. Phys. B: At. Mol. Opt. Phys. 211489

Lin C D 1984 Phys. Rev. A 291019

Lin C D, Macek J 1984 Phys. Rev. A 292317

Makrewicz J 1989 J. Phys. B: At. Mol. Opt. Phys. 22 L235

Pekeris C L 1958 Phys. Rev. 1121649

Poirier M 1989 Phys. Rev. A 403498

Richter K, Wintgen D 1990 J. Phys. B: At. Mol. Opt. Phys. 23 L197

Rost J M, Briggs J S 1988 J. Phys. B: At. Mol. Opt. Phys. 21 L233

1989 J. Phys. B: At. Mol. Opt. Phys. 223587

1990 J. Phys. B: At. Mol. Opt. Phys. 23 L339

Rost J M 1990 Thesis University of Freiburg unpublished (in German)

Rost J M, Briggs J S, Feagin J M 1991 Phys. Rev. Lett. 661642 
Sadeghpour H R, Greene C H 1990 Phys. Rev. Lett. 65313

Sinanoglu O, Herrick D E 1973 J. Chem. Phys. 62886

Watanabe S 1987 Phys. Rev. A 361566

Wintgen D 1991 to be published

Wulfman C, Sukeyuki K 1973 Chem. Phys. Lett. 23367

Yuh H J, Ezra G S, Rehmus P, Berry R S 1981 Phys. Rev. Lett. 47497 